\title{
Reconstrucción microquirúrgica inmediata tras faringolaringuectomía: 10 años de experiencia
}

\section{Immediate microsurgical reonstruction after pharyngolaringhectomy: 10 years experience}

\author{
Carrasco-López, C. ${ }^{*}$, Lopez-Ojeda, A.B. ${ }^{\star}$, Serra-Payro, J.M. ${ }^{\star *}$, \\ Palacín-Porte, J.A., Viñals-Viñals, J.M.***

Resumen

La cirugía reconstructiva tras faringolaringuectomía circular por carcinoma avanzado de faringe/laringe es de alta complejidad. Hasta el momento se han planteado diferentes opciones reconstructivas sin que se haya podido clarificar cuál es la mejor o determinar el protocolo a seguir en estos casos.

Presentamos 10 años de experiencia en este tipo de reconstrucciones (1998-2008). Analizamos 25 casos de reconstrucción microquirúrgica de faringe tras faringolaringuectomías por carcinoma avanzado y revisamos el estado actual del tema.

Se emplearon 15 yeyunos y 10 colgajos fasciocutáneos para reconstrucción de faringe tras faringolaringuectomía; el 83,3\% de los pacientes volvieron a tolerar dieta. En el grupo en el cual se realizó reconstrucción con yeyuno observamos una media de inicio de ingesta de 13 días, a diferencia del grupo no yeyuno que la inicio a los 27 días. Las diferencias entre ambos grupos no fueron significativas.

A pesar de que actualmente aún no se ha aclarado cuál es la mejor reconstrucción para estos casos, en nuestra experiencia preferimos el colgajo visceral debido a su baja morbilidad local así como a sus buenos resultados locales.

\begin{tabular}{|c|c|}
\hline Palabras clave & $\begin{array}{l}\text { Carcinoma de faringe, Carcinoma de laringe, } \\
\text { Faringolaringuectomía, Reconstrucción } \\
\text { faringe, Reconstrucción laringe, } \\
\text { Colgajo yeyuno, Colgajos fasciocutaneos. }\end{array}$ \\
\hline Nivel de evidencia científica & científica \\
\hline
\end{tabular}

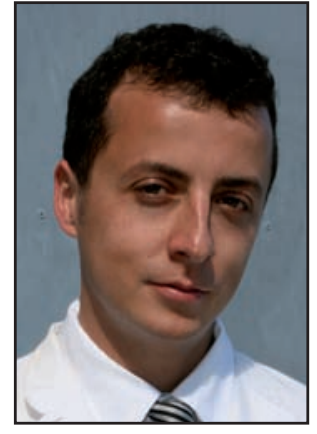

Carrasco López, C. 


\section{Introducción}

El carcinoma faringolaríngeo se diagnostica habitualmente en estadios avanzados y por tanto su pronóstico es limitado (1). Las principales razones de esta baja esperanza de vida son: su tardío diagnóstico, su infiltración únicamente submucosa y su rápida capacidad de metastatización (2).

En la actualidad, el tratamiento estándar es la cirugía con radioterapia o quimioterapia coadyuvantes (3). En la mayoría de los pacientes, la cirugía debe ser radical, mediante laringuectomía total o parcial con faringuectomía total y/o esofaguectomía. En una revisión de la literatura, solo el $15-34 \%$ de los pacientes cumple los criterios para una cirugía conservadora.

El defecto secundario tras la cirugía radical en el tratamiento del carcinoma de faringe/laringe avanzado provoca una disrupción del tránsito digestivo alto que debe reconstruirse siempre y, por tanto, supone un importante reto quirúrgico para el cirujano plástico. Históricamente, se han descrito múltiples procedimientos reconstructivos. El método ideal sería aquel capaz de reconstruir el defecto empleando un único procedimiento quirúrgico que además sea de de alta seguridad en la transferencia de tejidos, baja morbilidad en la zona donante, baja frecuencia de estenosis y/o fístula y, sobre todo, con capacidad de permitir de forma rápida en el paciente la rehabilitación de la habilidad para deglutir $(1,4)$.

El objetivo de este estudio es describir nuestra experiencia en reconstrucción de defectos primarios tras faringolaringuectomía circular y comparar el tiempo transcurrido hasta la rehabilitación de la deglución según el método reconstructivo empleado, al tiempo que realizamos una revisión del conocimiento actual sobre el tema.

\section{Material y método}

Llevamos a cabo una revisión retrospectiva de 10 años, entre 1998 y 2008, de la base de datos de historias clínicas del Hospital Universitario de Bellvitge, Barcelona, España, buscando pacientes intervenidos por los Servicio de Otorrinolaringología y Cirugía Plástica; analizamos un total de 25 reconstrucciones microquirúrgicas en pacientes con tumores de hipofaringe y laringe localmente avanzados. En todas las historias clínicas constaban claramente especificados los días trasnscurridos entre la cirugía y el inicio de la ingesta, así como las complicaciones postoperatorias. Todos los pacientes incluidos en este estudio habían sido diagnosticados de neoplasia de faringe y/o laringe avanzada tributaria de cirugía radical con reconstrucción inmediata.

Diseñamos 2 grupos: uno con aquellos pacientes a los que se les había realizado reconstrucción con colgajo yeyunal y otro con aquellos pacientes en los que se había realizado reconstrucción con colgajo fasciocutáneo. La elección del tipo de colgajo para reconstrucción se había hecho siguiendo los siguientes criterios: si no existían antecedentes de cirugía abdominal compleja se realizó reconstrucción con colgajo libre de yeyuno; si el paciente presentaba historia de cirugía abdominal compleja se realizó reconstrucción con colgajo fasciocutáneo (Fig. 1-3).

A su vez, hicimos entre ambos grupos un estudio descriptivo-comparativo de las siguientes variables: complicaciones agudas tanto en zona receptora como donante, tiempo de hospitalización, tiempo de injerto, complicaciones postoperatorias, así como recidiva y éxitus durante el seguimiento. La distribución de estas variables fue investigada mediante el test de Shapiro-Wilk.

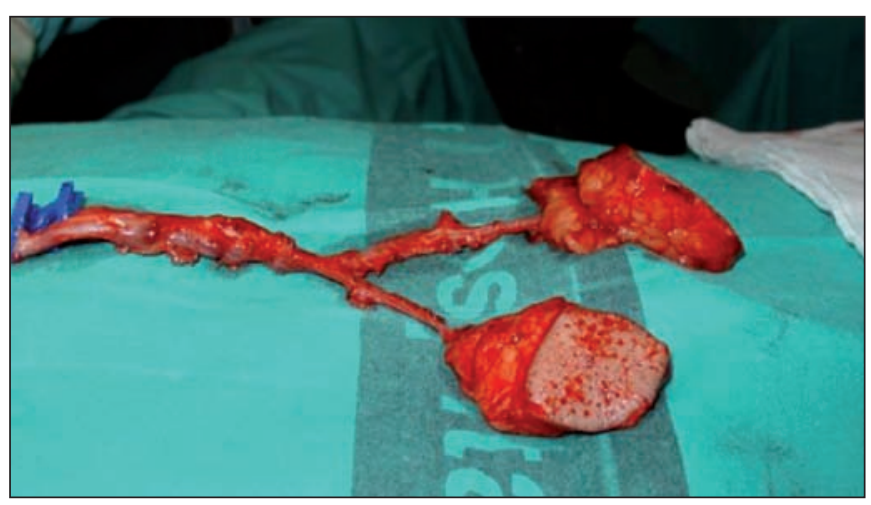

Fig. 1. Reconstrucción parcial de faringe y de piel por resección de ambas con colgajo ALT (anterolateral) doble isla.

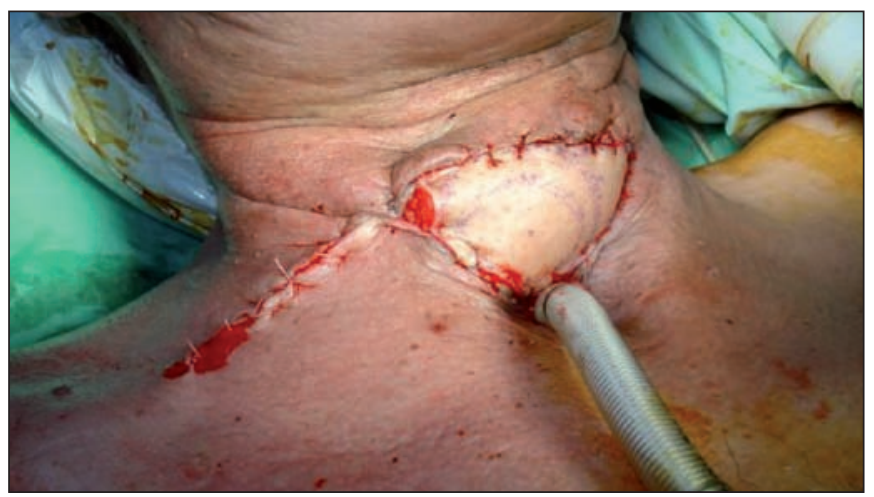

Fig. 2. Resultado inmediato de reconstrucción con ALT doble isla; visión de piel.

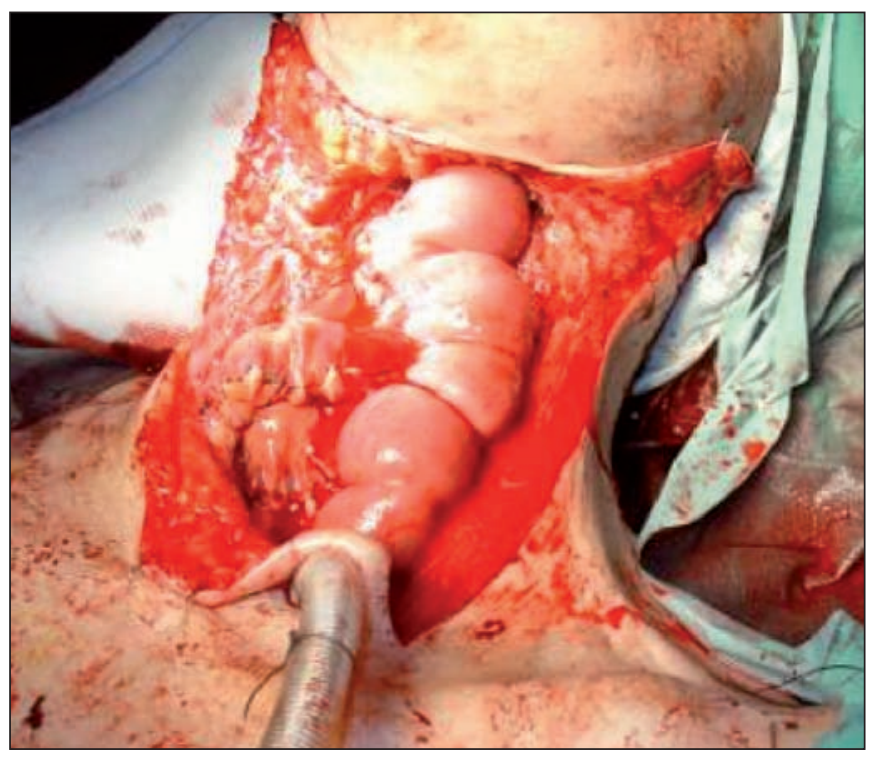

Fig. 3. Reconstrucción de faringuectomia total con yeyuno. 
Examinamos también los factores de riesgo que pudieran suponer diferencias importantes entre el grupo de pacientes con colgajo de yeyuno libre o con otros colgajos mediante los tests de Qui-cuadrada, t de student y Wilcoxon.

\section{RESULTADOS}

La edad media de los pacientes estudiados fue de 60,72 años (Desviación standard, DS, 9,67; 46-82 años), con un total de 22 hombres y 3 mujeres.

La localización de las neoplasias fue: 4 tumores de orofaringe, 16 de hipofaringe y 5 de laringe.

Las reconstrucciones se realizaron con colgajo yeyunal libre en 15 pacientes, y en otros 10 con colagjos fasciocutáneos: 8 radiales y 2 anterolaterales.

La supervivencia global de los colgajos realizados fue de 22 pacientes ( $88 \%, 1$ necrosis yeyunal y 2 radial) sin diferencias significativas $(\mathrm{p} 0,10)$ entre los dos grupos. Las causas de la necrosis se dividieron en 2 necrosis de colgajo radial por trombosis venosa y 1 necrosis yeyunal por trombosis arterial.

Las complicaciones agudas se distribuyeron en: 2 hematomas (todos en los colgajos de yeyuno); 7 fístulas ( 2 en colgajos anterolaterales, 4 en radiales, 1 en colgajo de yeyuno); 3 necrosis ( 2 en colgajos radiales y 1 en colgajo de yeyuno); y 1 dehiscencia yeyunal de pared abdominal que se trató con resutura de la pared (Gráfíca 1).

La frecuencia de complicaciones tardías (extrahospitalarias) se constató de la siguiente manera: 5 estenosis ( 3 en colgajos radiales y 2 en colgajos yeyunales) y 1 fístula crónica en un colgajo radial (Gráfica 1).

No observamos diferencias significativas en ninguno de los 2 grupos en el número y frecuencia de complicaciones (p 0,08$)$.

La media de la estancia hospitalaria fue de 31 días (rango intercuartilico, RIC 26,50). Aquí observamos que en los casos de colgajo yeyunal los días de estancia fueron como media 28 y en los casos de colgajo fasciocutaneo 34 días. A su vez, el inicio de la ingesta postcirugía tuvo una media de 15 días (RIC 23,50), no encontrando diferencias significativas entre los 2 grupos analizados

Gráfica 1.

Cuadro resumen de colgajos realizados, así como de sus complicaciones

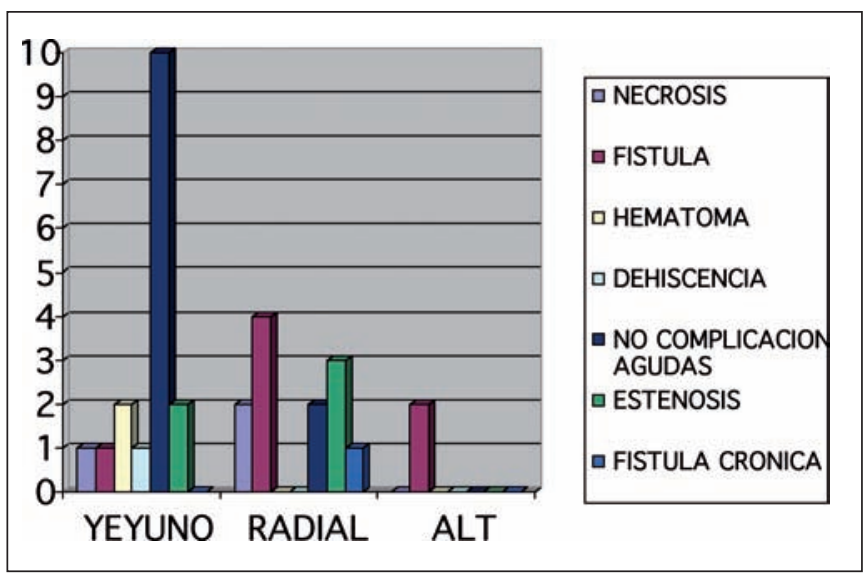

Gráfica 2.

Media de inicio de tolerancia oral a los 15 días

de postoperatorio

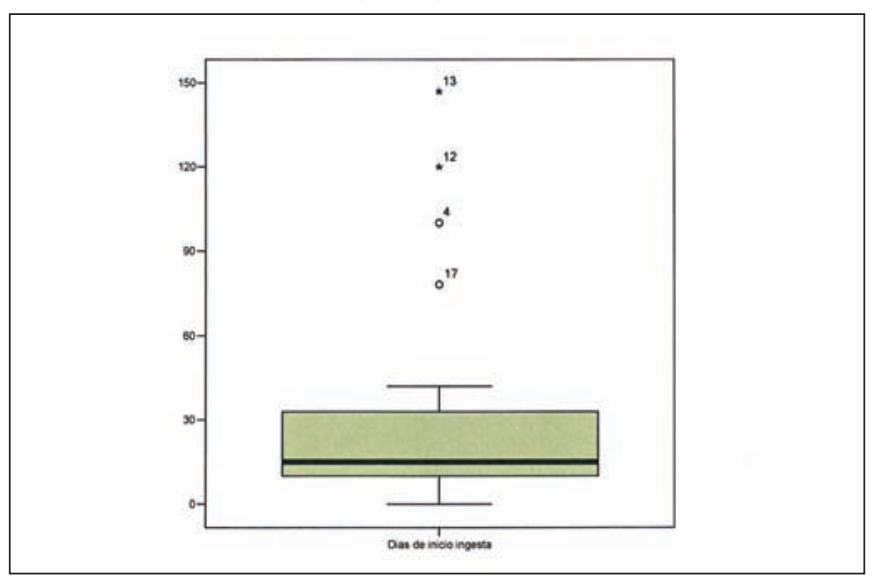

Gráfica 3.

Entre los dos grupos, a pesar de no existir diferencias significativas, observamos que en el colgajo 1 (yeyuno) el inicio de la dieta se produjo antes que en el grupo colgajo 2 (fasciocutáneos).

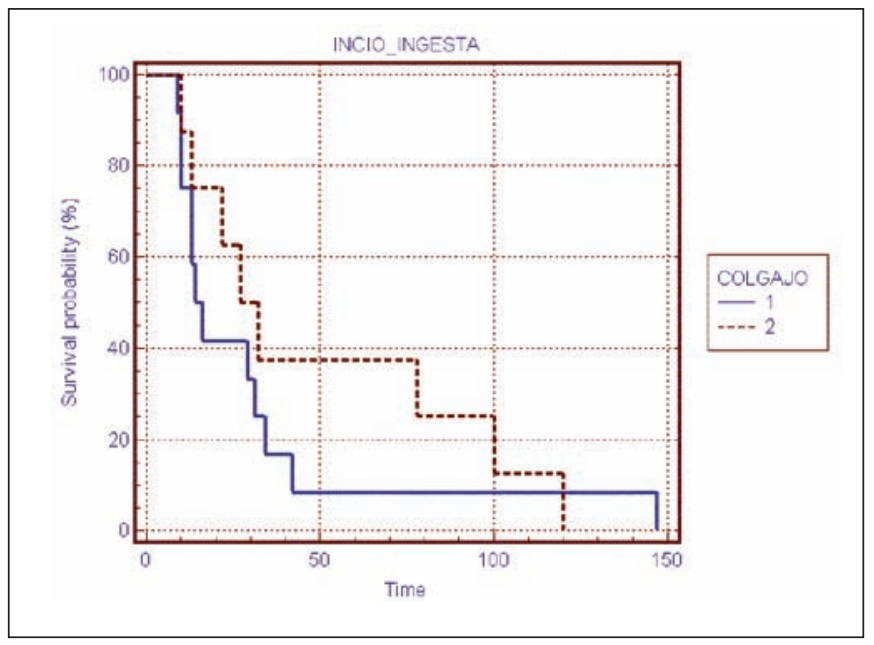

Gráfica 4.

Supervivencia muy similar en ambos grupos, con una evolución muy paralela. Colgajo 1 (yeyuno) y colgajo 2 (fasciocutáneos).

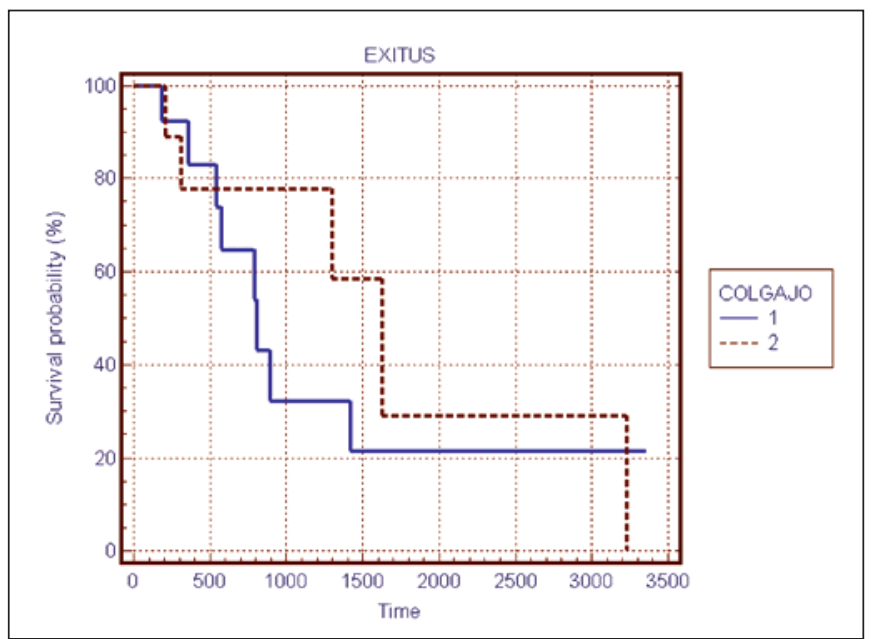

(p 0,06). Si observamos entre los diferentes grupos, vemos que en el grupo de colgajo yeyunal, el inicio de la dieta tuvo una media de 11 días y en el grupo de colgajos fasciocutáneos de 18 días (Gráfica 2) 
En nuestro estudio, 21 pacientes $(83,3 \%)$ volvieron a tolerar dieta. En total solo 4 pacientes no consiguieron tolerarla: 3 pacientes con colgajo radial y 1 con colgajo yeyunal (Gráfica 3).

La mayoría de los pacientes recibieron tratamiento coadyuvante: en 11 solo con quimioterapia y en 12 con radioterapia y quimioterapia.

Durante el seguimiento encontramos 9 pacientes (32\%) con recidivas locoregionales, sin observar diferencias significativas entre los 2 grupos $(\mathrm{p} 0,12)$. El periodo medio de recidiva de nuestro grupo de pacientes fue 499,89 días (DS, desviación estándar, 430,09). Otros 13 pacientes $(52 \%)$ fueron exitus. El periodo de media de supervivencia fue de 1002,17 días (con un máximo de 3230 días y un mínimo de 184 días, y sin diferencias significativas entre los 2 grupos (p 0,09) (Gráfica 4).

\section{Discusión}

El cáncer avanzado tanto de faringe como de laringe supone una enfermedad con mal pronóstico. El porcentaje de supervivencia a 5 años oscila entre el 25 y el $35 \%$. Por ello, los procedimientos realizados en estos pacientes, en general, tienen carácter paliativo (5).

Uno de los propósitos fundamentales del tratamiento quirúrgico es eliminar el tumor y lograr una rehabilitación deglutoria rápida y oportuna que permita al paciente recuperar su estado nutricional. Esto es importante para que así pueda exponerse a otros tratamientos tóxicos o recuperarse de los ya recibidos. Y todo ello debe lograrse antes de que recurra la enfermedad (6).

Desde que se instauró la microcirugía y la transferencia de colgajos libres, se han empleado muchos modelos de colgajos para la reconstrucción de importantes defectos postquiúrgicos, lo que ha aportado sin duda un gran progreso en el tratamiento de los pacientes, sobre todo en los casos de reconstrucción de defectos en cabeza y cuello $(7,8)$. No existe un acuerdo universal acerca de cuál es el método óptimo de reconstrucción microvascular. El colgajo radial, de yeyuno o el anterolateral, han probado ser técnicas útiles a pesar de sus desventajas. Por ejemplo, el colgajo yeyunal ofrece una minima morbilidad de la zona donante y un bajo porcentaje de fístulas. En cambio el colgajo radial puede ofrecer una mejor calidad en el lenguaje a expensas de un incremento del porcentaje de fístulas (4).

Según un estudio realizado por Takashi y col. en el que se compara la reconstrucción con colgajo de yeyuno y con colgajo radial de faringostomas, se demuestra que en la reconstrucción con radial aparecen de forma significativa más fístulas que con el colgajo de yeyuna. Esto provoca un retraso en el reinicio de la dieta oral en comparación a cuando se emplea la reconstrucción con colgajo yeyunal. Así mismo, también comprobaron más estenosis en el colgajo radial que en el grupo de colgajo de yeyuno (9).
Los defensores del grupo de colgajos fasciocutáneos se basan en una menor morbilidad de la zona donante, así como en una mejor rehabilitación del lenguaje. Los grupos que defienden el colgajo visceral presentan menos fístulas y menos estenosis que con los colgajos fasciocutáneos (10).

En nuestro estudio, no observamos diferencias significativas entre los 2 grupos; esto puede ser debido a presentar una muestra limitada. Sin embargo sí encontramos pequeñas variaciones. En el primer grupo (colgajo yeyunal) observamos una mejor evolución en cuanto al inicio de la ingesta, número de fístulas y estancia hospitalaria. Además, objetivamos también en este tipo de colgajo una tendencia hacia una baja morbilidad de la zona donante, así como a una más rápida recuperación del paciente en el inicio de la dieta y un menor número de complicaciones locales.

Por estas razones, y a pesar de no poder demostrar diferencias significativas entre los 2 grupos analizados, en nuestra experiencia, la preferencia en reconstrucciones tras faringolaringuectomías circulares es el colgajo visceral yeyunal (9).

\section{Conclusiones}

Podemos asumir que las indicaciones para la cirugía recosntructiva microquirúgica en faringolaringuectomía por carcinoma son muy estrictas. El tipo de colgajo escogido depende de las condiciones del paciente así como de la extensión del tumor y de la experiencia del cirujano.

A pesar de que en estos pacientes el pronóstico sigue manteniéndose muy pobre, la reconstrucción de faringe con colgajo libre ofrece una rápida rehabilitación y una razonable calidad de vida.

\section{Agradecimientos}

Agradecemos la colaboración del Servicio de Medicina Preventiva del Hospital de Bellvitge por parte de Cristina Masuet Aumatell, Andrea Juliana Sanabria Uribe e Isabel Román Romera, en la realización del estudio, cálculo y análisis estadístico.

\section{Dirección del autor}

Dr. Cristian Carrasco López

Servicio de Cirugía Plástica

Hospital de Bellvitge

Feixa Llarga, s/n

08907 Hospitalet de Llobregat

Barcelona, España

correo electrónico: carralo@gmail.com 


\section{Bibliografía}

1. Pen-Yuan Chu, Shyue-Yih Chang.: Reconstruction of the Hypopharynx After Surgical Treatment of Squamous Cell Carcinoma J Chin Med Assoc 2009; 72 (7): 351-355

2. Hwang Y, Chang SY, Hwu BC, Chang P.: The factors of diagnosis and treatment delay in the late stage of cancers in pharynx, larynx and oral cavity $J$ Chin Med Assoc 1992; 49:86-91.

3. Lefebvre JL, Chevalier D, Luboinski B, Kirkpatrick A, Collette L, Sahmoud T.: Larynx preservation in pyriform sinus cancer: preliminary results of a European Organization for Research and Treatment of Cancer phase III trial EORTC Head and Neck Cancer Cooperative Group. J Natl Cancer Inst 1996, 88: 890-899.

4. Disa J, Pusic A, Hidalgo D, Cordeiro P.: Microvascular Reconstruction of the Hypopharynx: Defect Classification, Treatment Algorithm, and Functional Outcome Based on 165 Consecutive Cases. Plast Reconst Surg. 2003. 111 (2): 652-660.

5. Benazzo, M; Occhini, A; Rossi, V.; Aresi, G, Alessiani, M.: Jejunum free flap in hypopharynx reconstruction: case series. BMC cancer. 2002. 2:13-18.
6. Garcia S; Binelfa F, Sánchez C.: Colgajo libre microvascularizado de yeyuno para reconstrucción faringoesofágica inmediata en cáncer faringolaríngeo avanzado. An ORL mex. 2006, 51 (2): 94-98.

7. Harii K, Ebihara S, Ono I, Saito H, Terui S, Takato T.: Pharyngoesophageal reconstruction using a fabricated forearm free flaps. Arch Otolaryngol 1982; 108: 303.

8. Nakatsuka T, Harii K, Yamada A, Asato H, Ebihara S.: Versatility of a free inferior rectus abdominis flap for head an neck reconstruction: analysis of 200 cases Plast Reconstr. Surg 1994; 93: 762-769.

9. Nakatsuka T; Harii K, Asato H, Ebihara S, Yoshizumi T, Saikawa M.: Comparative Evaluation in pharyngo-oesophageal reconstruction: radial forearm flap compared with jejunal flap. A 10-year experience. Scand. J PLast Reconstr Hand Surg. 1998. 32: 307-310.

10. Azizzade A; Sherry Y, Abemayor, E.: Radial forearm Free flap pharyngoesophageal reconstruction. The laryngoscope. 2001,111(5):807-810.

\title{
Comentario al artículo “Reconstrucción microquirúrgica inmediata tras laringolaringuectomía: 10 años de experiencia"
}

\author{
Dr. Eric Santamaria \\ Profesor de Microcirugía Reconstructiva, Universidad Nacional Autónoma de México. \\ Médico Adscrito al Servicio de Cirugía Plástica y Reconstructiva del Hospital General Dr. Manuel Gea González e Insti- \\ tuto Nacional de Cancerología. Ciudad de México, México
}

En este artículo se presenta la experiencia de 10 años con el uso de colgajos libres en la reconstrucción inmediata de defectos de faringe y esófago cervical secundarios a la resección de tumores malignos. Los autores comparan los resultados obtenidos con el uso de yeyuno en 15 pacientes y de colgajos fasciocutáneos en 10 pacientes: radial de antebrazo en 8 y anterolateral de muslo en 2 . El porcentaje de supervivencia de los colgajos y de éxito funcional en ambos grupos de pacientes fue muy satisfactorio.

Los autores describen que para seleccionar el tipo de colgajo utilizado tomaron como único criterio el antecedente de cirugía abdominal previa. Es cierto que cuando existe historia de laparotomía exploradora y obstrucción intestinal por bridas, está contraindicada la utilización del yeyuno. Sin embargo, en pacientes sin bridas intestinales, es posible disecar un colgajo libre de yeyuno sin incremento de la morbilidad.

Otros aspectos que no se mencionan en el artículo y que son críticos para elegir el tipo de colgajo que se debe usar y evaluar los resultados funcionales, son el tamaño y tipo de cada defecto, si recibieron radioterapia previa a la reconstrucción y cuáles fueron los vasos sanguíneos receptores para los colgajos libres $(1,2)$; pues en pacientes en los que se realiza una disección radical de cuello bilateral y/o con historia de radioterapia preoperatoria, muchas veces no contamos con vasos sanguíneos fiables o cercanos al defecto para anastomosar un colgajo libre de yeyuno, que tiene un pedículo muy corto, o requerimos de injertos venosos. Mientras que la presencia de un defecto cutáneo en el cuello asociado al defecto en esófago obliga a usar dos islas de piel del mismo colgajo (como ilustran los autores en un caso), la utilización de dos colgajos libres o de un colgajo cutáneo regional simultáneo al yeyuno, para reconstruir tanto el defecto del tracto digestivo superior, como el defecto cutáneo.

A pesar de que los resultados en cuanto a la supervivencia del colgajo fueron muy similares en ambos grupos, hubiera sido interesante saber cómo llevaron a cabo la monitorización postoperatoria de los colgajos libres y cuánto tiempo después de la reconstrucción se presentó la trombosis arterial o venosa en los colgajos complicados. Debido a que estos son colgajos ocultos, si no contamos con un monitor externo que nos permita detectar tempranamente una trombosis, la posibilidad de poder salvar un colgajo fallido disminuye significativamente. En particular el de yeyuno, que tiene poca tolerancia a la isquemia. La permeabilidad de los colgajos ocultos puede hacerse con un doppler implantable, o cuando no se cuenta con este método, es recomendable agregar un segmento del yeyuno como monitor externo y retirarlo 5 o 6 días después de la reconstrucción, bajo anestesia local (Fig. 1).

Al final, los autores concluyen (al igual que en otros estudios), que el colgajo de yeyuno sigue siendo la primera opción 
para reconstrucción de defectos postlaringo/ faringuectomía y sin duda lo es, sobre todo en resecciones extensas de la hipofaringe y del esófago cervical $(1,2)$. El uso de un colgajo visceral cumple el principio básico de reconstruir el defecto, siempre que se pueda, con el mismo tejido. El hecho de tener que formar un tubo circular a partir de una isla de piel poco elástica, implica agregar una línea de sutura longitudinal adicional a las anastomosis proximal y distal al defecto reconstruido, lo cual incrementa el riesgo de fístula y/o estenosis y retrasa significativamente el inicio de la alimentación por vía oral, tal y como describen en este trabajo el Dr. Carrasco López y col.
Bibliografía

1. Cordeiro, P.G., Shah, K., Santamaria, E., et al.: Barium swallows after free jejunal transfer: Should they be performed routinely? Plast Reconstr Surg 1999. 103: 1167.

2. Disa J, Pusic A, Hidalgo D, Cordeiro P.: Microvascular Reconstruction of the Hypopharynx: Defect Classification, Treatment Algorithm, and Functional Outcome Based on 165 Consecutive Cases. Plast Reconst Surg. 2003. 111 (2): 652-660.
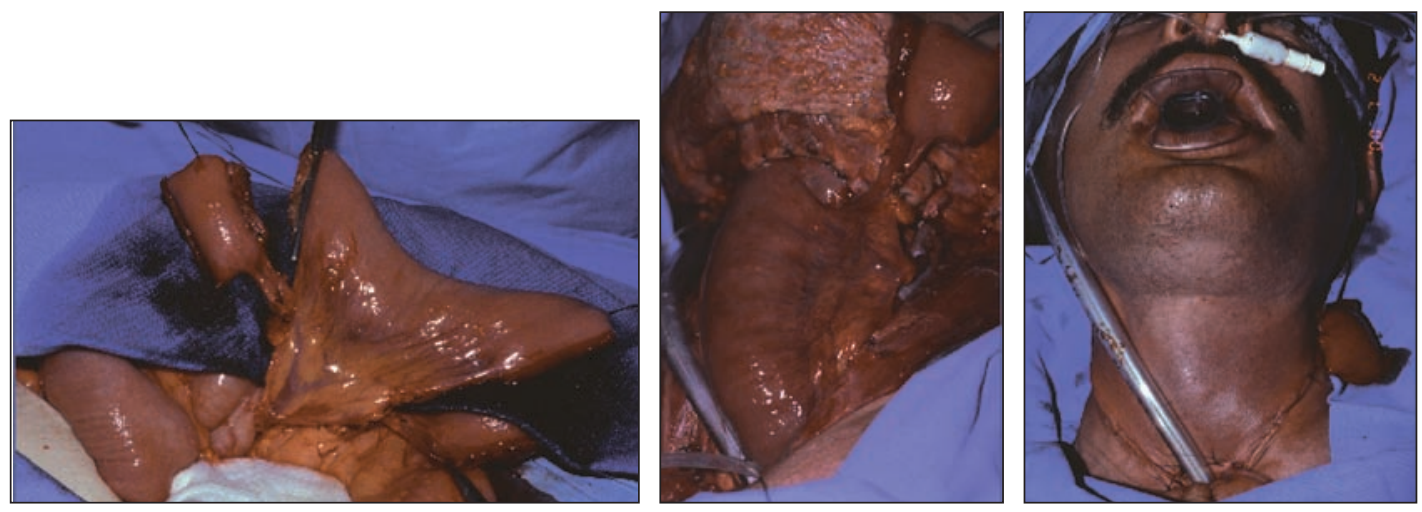

Fig. 1. Reconstrucción de defecto esofágico cervical con colgajo libre de yeyuno. Junto con la disección del colgajo se incluye un segmento centinela que se exterioriza para monitorlzar la vascularización del colgajo. Éste se retira al cabo de 5 a 6 días bajo anestesia local.

\section{Respuesta al comentario del Dr. Santamaría}

\section{Dr. Cristian Carrasco López}

Agradecemos al Dr. Santamaría sus comentarios a nuestro trabajo, así como sus observaciones y recomendaciones.

Es muy interesante su aportación sobre la disección de un colgajo libre de yeyuno a pesar de cirugía abdominal previa. Nuestra experiencia es que hemos realizado colgajos viscerales con éxito en pacientes con cirugías previas. Sin embargo a la hora de planificar la cirugía siempre considerábamos la posibilidad de fracaso en estos casos.

En estos pacientes, es un punto crítico escoger los vasos receptores. Existe un gran número de publicaciones sobre el tema (1). En nuestra experiencia, cuando los vasos comunes cervicales (facial, tiroidea, carótida externa, etc.) no son suficientemente buenos, realizamos un injerto de vena y buscamos segundas opciones, como la arteria cervical transversa, y en otros casos la arteria mamaria interna (2).

En nuestro caso, la monitorización siempre se realizó con centinela externo, ya fuera cutáneo en el caso de los colgajos fasciocutáneos, o de grasa mesentérica en el caso yeyunal. 